\title{
ADAPTATION OF ILEAL EXPRESSION OF EPIDERMAL GROWTH FACTOR RECEPTOR IN GROWTH RESTRICTED NEONATAL PIGLETS - IMPLICATIONS FOR CATCH UP GROWTH?
}

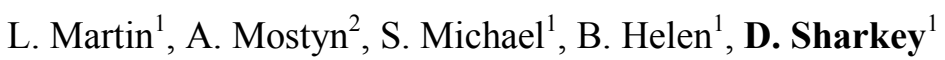 \\ ${ }^{1}$ Division of Academic Child Health, ${ }^{2}$ School of Veterinary Medicine and Science, University of Nottingham, \\ Nottingham, UK
}

Background: Epidermal growth factor (EGF) is present in breast milk and stimulates the proliferation and maturation of the gut. Growth restricted infants with postnatal catch up growth are at increased risk of obesity and the metabolic syndrome. The porcine litter offers a naturally occurring range of normally grown (NG) and growth restricted (GR) offspring.

Aims: Examine the mRNA abundance of EGF receptor (EGFr) in the ileum of NG and GR neonatal piglets.

Methods: NG and GR piglets were maternal fed. At 7 and 14 days postnatal age, piglet ileal mRNA expression of EGFr was quantified using real-time PCR.

Results: GR piglets had a significantly lower birth weight than NG $(\mathrm{P}<0.05)$. At 7 days of age, GR piglets ileal EGFr was significantly less than NG $(\mathrm{P}<0.05$, see figure) but there was no difference at 14 days of life when the GR had also achieved a similar weight to the NG. Intestinal weight at 7 and 14 days of age correlated with body weight $\left(\mathrm{R}^{2}=0.93\right.$ and 0.59 , respectively).

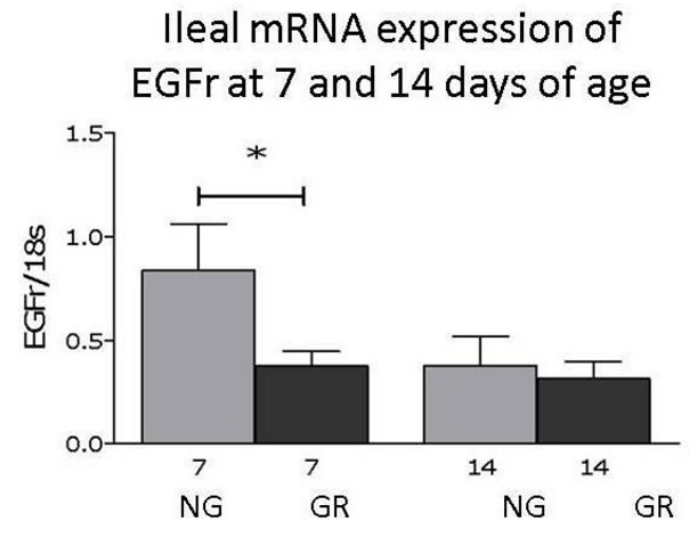

[Figure]

Conclusion: In a naturally occurring porcine model of growth restriction, EGFr is reduced at 7 days of life and normalises by day 14 coincident with a period of catch up growth. Accelerated intestinal maturation in the neonatal period may predispose growth restricted newborns to later risk of obesity and the metabolic syndrome. 